\title{
A Unified Handheld Terminal for WSN Data Gathering
}

\author{
Siquan $\mathrm{Hu}^{1, \mathrm{a}^{*}}$, Juan $\mathrm{Hu}^{1, \mathrm{~b}}$ and Chundong She $\mathrm{S}^{2, \mathrm{c}}$ \\ ${ }^{1}$ School of Computer and Communication Engineering, University of Science and Technology \\ Beijing, Beijing, 100083, China \\ ${ }^{2}$ School of Electronic Engineering, Beijing University of Posts and Telecommunications, Beijing, \\ 100876, China \\ ahusiquan@ustb.edu.cn, bhuj_ustb@163.com, shurcd@vip.sina.com
}

\begin{abstract}
Keywords: Handheld Terminal, WSN, Android, XML
Abstract. With the rapid development of wireless sensor network (WSN), the WSN data gathering is attracting more interests over the years. Due to the high cost of specialized handheld terminals, Android tablets are chosen to design a unified handheld terminal for WSN data gathering. Data are transmitted from WSN sink node to the Android terminal via Bluetooth. An Android application parses WSN data through XML file defined by users, and then stores the parsed WSN data into SQLite database, finally three functions are provided to observe the WSN data in real time. With XML technology, the system can separate data parsing from the application logic, thus can parse data for different data formats or WSN platforms with high flexibility.
\end{abstract}

\section{Introduction}

Recent advances on micro-electro-mechanism systems (MEMs) technology, wireless communications and digital electronics have enabled the wireless sensor networks (WSN) came into being $[1,2,3]$. WSN is loaded with an enormous amount of sensor data from real physical environment, thus data gathering is one of the most important aspects of WSN and this topic has witnessed significant amount of research over the years [4,5]. Nowadays, there are various handheld terminals which have a fancy price and poor generality on the market. As a result of these factors, this paper chose Android tablets, which are cheap and convenient to use and carry, to design a unified handheld terminal for WSN data gathering.

This unified handheld terminal can monitor several environmental parameters such as ambient temperature, atmospheric humidity, illumination and so on, which is entirely dependent on the XML file configured by users. Whenever the hardware, which is used to gathering WSN data, is changed for gaining different environmental parameters, all we have to do is to change the XML file of the handheld terminal so that it can still run as normal with full function and no exception, it's unified.

\section{Overall Design}

In this design, Android is chosen as the development platform of unified handheld terminal, with XML parsing technology applied to configure the software, Bluetooth serial ports adopted to transmit data, SQLite database utilized to store the WSN data, finally, end users can manage the WSN data in real time.

As is shown in Fig.1, the handheld terminal software is classified into four parts:

I When the software is started, the XML files configured by users will be parsed, and there will be a parsing result according to the parse.

I A data transmission will be created between Android device and WSN central node via Bluetooth serial ports communication, through which the WSN data packet will be gathered.

I With the parsing result and gathered data packet, the WSN data will be parsed and stored into the SQLite Database.

I Through reading data from the database, the monitoring and management of gathered WSN data is realized, three services are provided for end users: Data Analysis Table, Network Topology, and Data Line Graphs. 


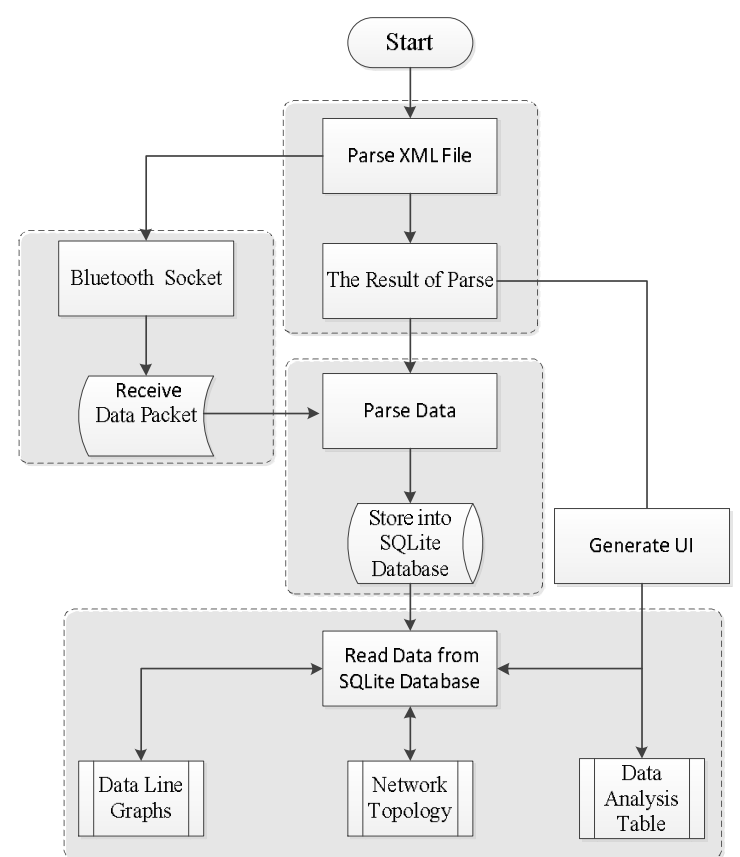

Fig. 1 The WSN Terminal Software Flow Chart

\section{Realization Process}

Four parts are introduced in this section: Configuration Module, Transmission Module, Storage Module and Service Module.

Configuration Module: XML File Parsing. A structured WSN packet is defined in a XML file, the parsing logic is also defined here. A sample of XML file as follows:

$<$ XFields $>$

$<$ Field $>$

$<$ Name name $=$ "inTemp" length length $=" 2$ " offset $=" 28 "><$ Name $>$

$<$ Conversion function=" $422.5^{*} x / y-277.7^{\prime \prime}>$

$<$ Param varName $=" x$ " varValue $=$ "inTemp" $><$ Param $>$

$<$ Param varName $=" y$ " varValue $=" v$ Batt" $>\quad</$ Param $>$

$</$ Conversion $>$

$</$ Field $>$

$<$ XFields $>$

The tag $<$ Field $>$ refers to the related contents of parameters which users need to extract from the WSN monitoring area, in which the tag $<$ Name $>$ stands for the meaning of parameters in the data packets, including the name of parameters(name), the length of data(length), and the position of data interception (offset). The tag $<$ Conversion $>$ includes the data processing function(function), in which tag $<$ Param $>$ consists of the name of function variable (varName) and the value of function variable (varValue). The XML file can be changed by add or reduce the $<$ Field $>$ tags all depending on the user's needs, which makes the developed handheld terminal extendible, reusable and unified. The result of XML file parsing is a ArrayList $<$ XMLField $>$, in which there are XMLField instances containing related contents of parameters such as name, length, offset, function and so on. With the parsing result, the data packets could be parsed on needs.

Transmission Module: Bluetooth Serial Port Communication. Bluetooth is selected on our unified handheld terminal's data transmission, that firstly the data of environmental parameters in the monitoring area is gathered to the sink node through WSN which consists of sensor nodes, then transmitted to the intelligent terminal via Bluetooth communication module, for users to operate and process on the devices. Its structure is showed in Fig. 2. 


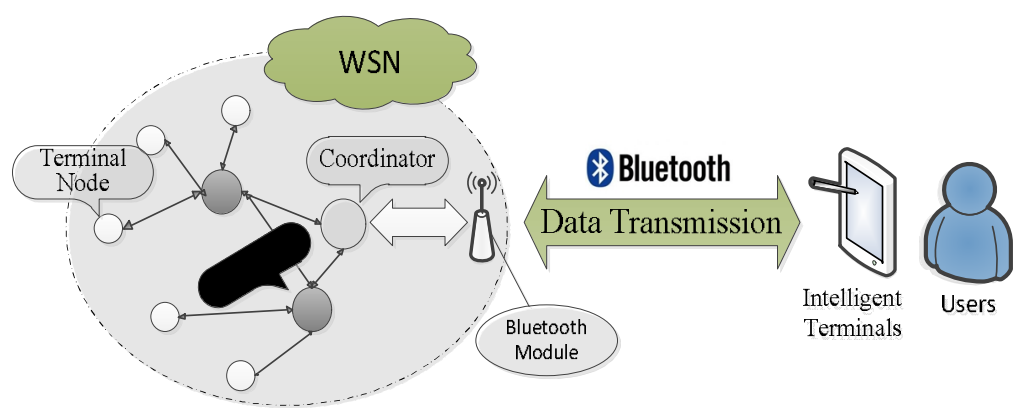

Fig.2 Data Transmission Structure

Storage Module : SQLite Database. The Unified handheld terminal applies SQLite database to store and manage the gathered data. SQLite is a lightweight class database, especially suitable for the CURD (create, update, read, delete) of a small amount of data, which supports most SQL grammars and allows using SQL language to operate the database. We store the received data into SQLite Database depending on the ArrayList $<$ XMLField $>$ too. Thus the store of received data is completely determined by the XML files defined by users.

Service Module: Real-time Data Display. The ultimate function provided to users by the Unified handheld terminal is to receive the data gathered from WSN via Bluetooth and display the real-time data on the user interface, which enables users to manage the data of WSN detecting area in real time.

Data Analysis Table. This function is to display all the received data in a table which is stored in the database after parsed. For more direct and clear display, the first row in the table is fixed in this design, while the rest uses scrollable page mode, as is shown in Fig.3.

\begin{tabular}{|c|c|c|c|c|c|c|c|c|c|c|c|}
\hline source & \multicolumn{2}{|c|}{ segno } & metric & parent & & Batt & \multicolumn{2}{|c|}{ inTemp } & humudity & \multicolumn{2}{|r|}{ temp } \\
\hline 0001 & & & $00 A 8$ & 0000 & & 9.2676 & 25.1 & & 47.141426 & & 29.2984 \\
\hline 0006 & & & 0072 & 0000 & & 9.2676 & 30.3 & & 45.030792 & & 30.6312 \\
\hline source & seqno & metric & parent & vBatt & inTemp & humidity & temp & light & MagX & MagY & Magz \\
\hline 0003 & 0000 & 0016 & 0000 & 2999.3 & 29.4 & 50.3 & 26.5 & 2586 & 2843 & 3100 & 3357 \\
\hline 0002 & 0000 & 0016 & 0000 & 2999.3 & 28.0 & 50.8 & 26.6 & 2586 & 2843 & 3100 & 3357 \\
\hline
\end{tabular}

Fig.3 Data Analysis Table

Network Topology. This function displays the network topology according to the relationship between parent nodes and sub nodes in the database, which makes it convenient for users to observe the dynamic situation of WSN. The network topology is shown as Fig.4.

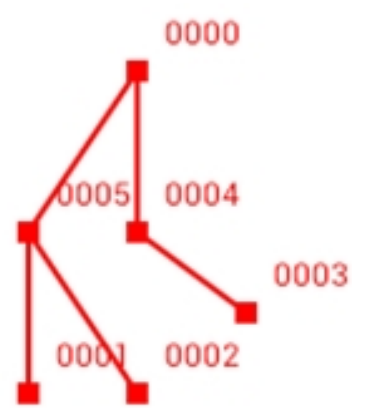

Fig.4 Network Topology

Data Line Graphs. This function displays various parameters of each node by line graphs, which is more intuitive for users. For example, we chose humidity, its history of received data will be presented in Fig.5.Every time a data packet is received via Bluetooth, the data history will refresh automatically with new data points added in the line graph. 


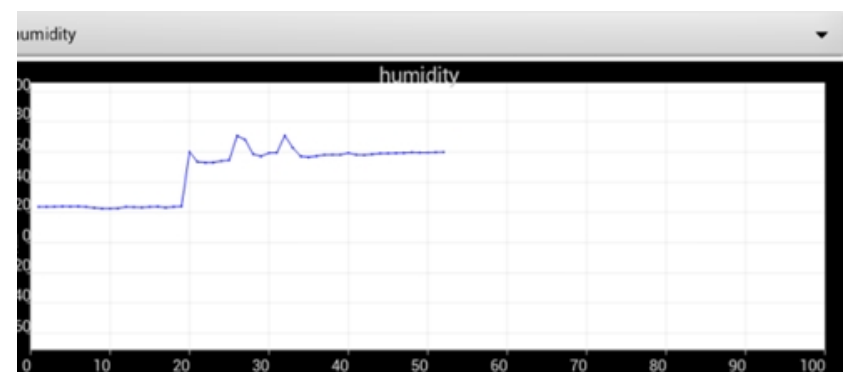

Fig.5 Data History

\section{Conclusion}

Based on Android platform and XML technology, a unified handheld terminal for data gathering in WSN is developed in this paper. We chose Bluetooth to transmit WSN data, parse data through XML file defined by users, store the parsed data into SQLite Database, provide data history, network topology and data analysis GUI to users. The flexibility is provided by that when the XML files are changed for monitoring more environmental parameters, the handheld terminal can still run as normal with full function and no exception.

\section{Acknowledgements}

The study was supported by the National Natural Science Foundation of China (No. 91438120) and the Foundation of Key Laboratory of Aerospace Broadband Network Technology.

\section{References}

[1] Boulmalf M, Belgana A, Sadiki T, et al. A lightweight Middleware for an e-Health WSN based System using Android Technology[C]. ICMCS 2012. IEEE, 2012: 551-556.

[2] Sankaranarayanan S, Wan A T. ABASH-Android based smart home monitoring using wireless sensors[C]. 2013 IEEE Conference on CEAT. IEEE, 2013: 494-499.

[3] Cardei M, Marcus A, Cardei I, et al. Web-based heterogeneous WSN integration using pervasive communication[C]. IEEE 30th IPCCC. IEEE, 2011: 1-6.

[4] Moreira N, Venda M, Silva C, et al. Sensor-Mobile application to monitor a WSN[C]. Information Systems and Technologies (CISTI), 6th Iberian Conference on. IEEE, 2011: 1-6.

[5] Ye D, Gong D, Wei W. Application of Wireless Sensor Networks in Environmental Monitoring[J]. Administration \& Technique of Environmental Monitoring, 2009, 1:205 - 208. 